\title{
MEASUREMENTS AND SIMULATIONS OF WAVE PROPAGATION IN AGITATED GRANULAR BEDS
}

\author{
Stephen R. Hostler and Christopher E. Brennen
}

\author{
Department of Mechanical Engineering, California Institute of Technology, Pasadena, CA 91125
}

\begin{abstract}
Wave propagation in a granular bed is a complicated, highly nonlinear phenomenon. Yet studies of wave propagation provide important information on the characteristics of these materials. Fundamental nonlinearities of the bed include those in the particle contact model and the fact that there exists zero applied force when grains are out of contact. The experimental work of Liu and Nagel showed the strong dependence of wave propagation on the forming and breaking of particle chains. As a result of the nonlinearities, anomalous behavior such as solitary waves and sonic vacuum have been predicted by Nesterenko. In the present work we examine wave propagation in a granular bed subjected to vertical agitation. The agitation produces continual adjustment of force chains in the bed. Wave propagation speed and attenuation measurements were made for such a system for a range of frequencies considerably higher than that used for the agitation. Both laboratory experiments and simulations (using a two-dimensional, discrete soft-particle model) have been used. The present paper is a progress report on the simulations.
\end{abstract}

\section{INTRODUCTION}

The complexities of wave propagation in a granular material are well documented. [1,2] Experiments have shown the sensitivity of wave propagation to the state of the material's microstructure which could even be changed by the wave itself [2]. Theoretical studies have predicted the existence of solitary waves and other interesting phenomena in these highly nonlinear materials [1]. Experiments have verified the existence of solitary waves in particle chains by comparing observed wave shapes to those predicted by the theory [3]. Despite the complexities, wave propagation in granular materials warrants further study as it is relevant in both industrial and natural settings and it also provides a promising, minimally invasive means of detecting buried objects [4] or investigating the state of the material itself.

Previous numerical studies have examined the properties of waves in systems with minimal disorder such as 1-D particle chains or 2-D regular packings. For a 2-D system, solitary waves have be observed to have very little dispersion while propagating vertically through the bed [4]. Other work focused on the depth dependence of the wave speed in a 2-D, triangular lattice [5]. In this paper, we look at the effect of irregularity in particle packing on the characteristics of wave propagation. Our simulations include dissipation so the waves can only be considered semi-permanent.

This paper will exclusively focus on simulations with pulse inputs. Simulations have also been run with continuous (sinusoidal) excitation. Results from these simulations display many qualitative features seen in our experimental investigation of $3-\mathrm{D}$ wave propagation. In particular, the 2-D simulations show the nonlinear beating and signal structure seen in the agitated bed experiments [6].

\section{SIMULATIONS}

The simulations are based on the discrete element method [7] in which each individual particle is tracked and its motion is determined by integrating the equations of motion. Interactions 
between particles are determined via a contact model that dictates normal and tangential forces due to contacts or collisions. We use a contact model that has a nonlinear, Hertzian spring in parallel with a viscous dashpot in the normal direction and a linear spring and frictional sliding element in the tangential direction [8]. As usual, the Hertzian spring yields the following non-linear relation between the normal force, $\mathrm{F}$, and the compression, $\delta$, of the particle of the form $\mathrm{F}=\mathrm{K}_{2} \delta^{3 / 2} .(\delta>0)$ and $\mathrm{F}=0 \quad(\delta<0)$. The dashpot and frictional slider introduce a mechanism of energy dissipation in the system.

The computational domain consists of a rectangular, 2-D cell with fixed walls on the top and right side and movable walls at the bottom and left side. All simulations were performed in the presence of gravity. The box contains a few thousand particles that are initially randomly placed but settle into a packed bed during a preliminary phase of the calculation. A pulse is then initiated by movement of the left wall and, in some cases, movement of the floor is used to agitate the bed. The excitation introduced at the left wall is measured at the right wall by one or more detectors of specified size and depth in the bed. This procedure emphasizes the influence of disorder in packing and agitation on wave propagation by creating a unique microstructure for each test.

The pulses consist of a period of constant positive acceleration followed by an equal portion of the negative of this acceleration. This pulse shape has the advantage of not including any infinite acceleration (and corresponding infinite force) whereas a pulse in velocity or displacement would apply such a force. Some simulations were run with other pulse shapes. The propagating pulse shapes showed little dependence on the shape of the input pulse.

The simulation variables were nondimensionalized using the mean particle diameter, the mean particle mass, and $(E / \rho)^{1 / 2}$ where $E$ and $\rho$ are the Young's modulus and density of the particle material. This scaling leads to a formulation in which the main parameters are a Froude number, the coefficient of restitution, Poisson's ratio, sliding friction coefficients, the number of particles, and geometric factors. In the contact model, this scaling reveals that the non-dimensional

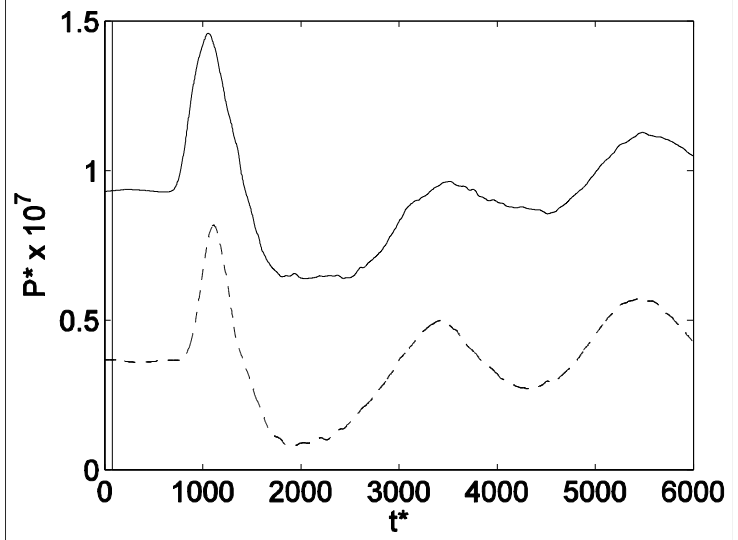

FIGURE 1. Waves shapes measured at two depths in a bed that is 50 particle diameters wide. The solid and dashed lines correspond to a sensor centered at 56 and 26 particle diameters below the free surface, respectively.

multiplying factor in the nonlinear normal spring relation is $\mathrm{K}_{2}^{*}=2 / \pi\left(1-v^{2}\right)$ and is only a function of Poisson's ratio.

\section{RESULTS}

\section{Static Bed}

A typical received signal is shown in Fig. 1 for a case in which the ratio of the total pulse displacement to the static deformation of a particle is of the order of ten. The first peak is the semipermanent wave with the subsequent peaks corresponding to its reflections off of the cell walls. The duration of the input pulse for this case is 80 time units as shown at the left side of figure. This comparatively short pulse leads to a wave with a width roughly ten times larger. Further simulations show that this wave length is quite insensitive to simulation parameters including the duration, amplitude, and shape of the input pulse.

A similar simulation with $10 \mathrm{x}$ the input pulse duration also produces a wave with a width of around 1000 time units. These periods correspond to a pulse width of 40 particles which is much larger than the $\sim 5$ particle diameters predicted by theory for the width of a solitary wave in a particle chain [1].

Despite the noticeable attenuation, the wave appears to maintain its shape through the reflections suggesting that the wave may be a solitary wave. 


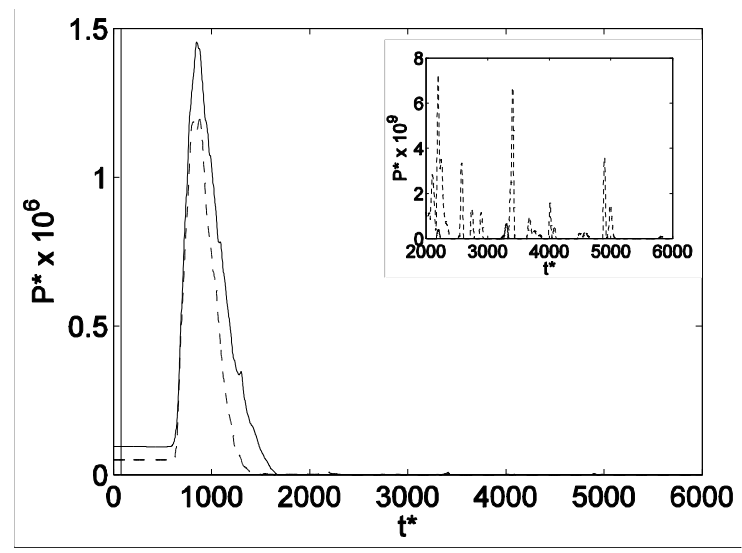

FIGURE 2. Wave shapes with $10 x$ the acceleration input. The solid and dashed lines correspond to a sensor centered at 56 and 26 particle diameters below the free surface, respectively. The inset is a vertically magnified view of the collisional behavior for $t^{*}>2000$.

Early calculations tend to show the velocity of the wave increases slightly as it traverses the box. This may be explained in that the wave is still forming over the relatively small size of the box.

The received signal for a case with ten times the input pulse acceleration is shown in Fig. 2. The pulse duration is the same as before and again displayed at the left of the figure. The period of the waves is nearly identical to that in Fig. 1, but the leading edges here appear to be steeper. The steepening of the wave front is indicative of a shock-like structure such has been observed in simulations of an elastic wave propagating vertically in a granular bed [9]. Additionally, there are no reflections seen as the pressure bottoms-out behind the wave. The expansion wave that follows the compression wave appears to pull the particles out of contact with the right walls, disrupting contact with the detectors. This theory is reinforced by the insert plot in Fig. 2 that shows a close-up of the wake region. The multiple peaks in this plot have durations that correspond to the contact time of a collision of a single particle with the wall. This region behind the compression wave displays collisional behavior until lasting contact is restored.

Figure 3 shows the propagation of the wave in the bed in more detail. Each of the particles are plotted with the net force on the particle in the horizontal direction represented by the shading. Black corresponds to zero force, white corresponds
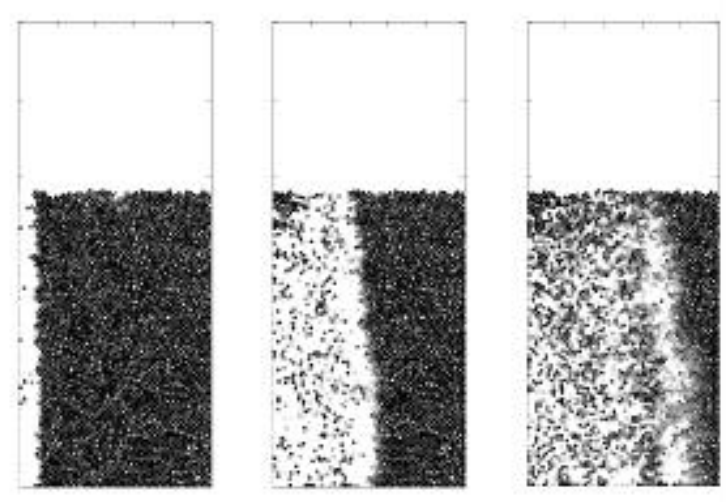

FIGURE 3. The wave front as it propagates across the bed. The particles are shaded according to the magnitude of the force on the particle in the $\mathrm{x}$-direction. The time between frames is 300 time units. The cell is 50 particles wide and the free surface is at a height of 76 particles.

to maximum force. The wave front, seen as the white band in the first frame, is relatively flat just after the pulse has been applied. By the time the wave has traveled halfway across the box, there is already quite a bit of curvature to the wave front. The wave moves faster deeper in bed due to the dependence of the wave speed on pressure. Disorder in the packing of the bed leads to some corrugation of the front, but, in general, the trend is that of increasing velocity with depth. This disorder coupled with dissipation leads to a less sharply defined front by the third frame. Judging by Fig. 1, despite the apparent spreading in Fig. 3, the shape of the wave seems to remain coherent. This spreading of the wave front may actually be the process of formation of the semi-permanent wave. Even with the less clearly defined front, the curvature is still evident in the third frame.

\section{Agitated Bed}

Here we will show some typical results from the simulation with floor agitation. Since the width of the pulse is much shorter than the period of the vertical shaking, the pulse is able to probe the state of the bed at an instant in time.

For the wave depicted in Figs. 4 and 5, the base of the bed has just passed through its highest elevation and is on its way to its mean agitation position as shown in the insert of Fig. 5. At this point on its trajectory, one would expect the bed to 

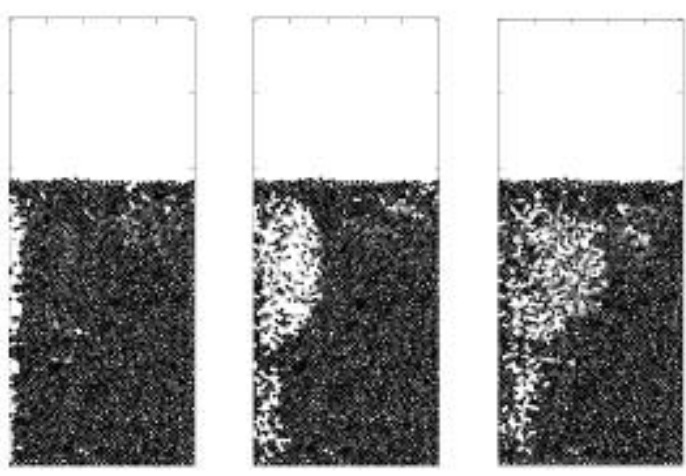

FIGURE 4. Pulse sent through an agitated bed in which the floor acceleration is $\pm 1.5 \mathrm{~g}$. The particles are shaded by the magnitude of the force on the particle in the $\mathrm{x}$ direction.

be expanding from the bottom. An expansion wave starts at the bottom and propagates to the top of the bed. Figure 4 shows the pulse passing through the top half of the cell where the particles are still in contact. In the lower half of the bed, the particles have come out of contact and the pulse cannot be transmitted. This description is corroborated by looking at the wave shapes in Fig. 5. The upper sensor detects a wave of similar shape to those seen in the static cases. A couple of waves that may have followed a slightly longer path obscure the back edge of the wave, but the width of the primary peak is consistent with the widths seen in Figs. 1 and 2. As for the sensor located 56 particle diameters from the free surface, no wave is measured at all.

The wave speed measured in the top section is $30 \%$ lower than that measured for an identical static case, suggesting that, though the particles are in contact, they are held by a much weaker confining force. This is supported by the much lower static pressure seen in Fig. 5 as compared to Fig. 1.

\section{DISCUSSION AND CONCLUSIONS}

We observe some form of semi-permanent wave that has a fixed width of roughly 40 particle diameters over large ranges of system parameters. Neglecting attenuation, the waves appear to maintain their shape, but show some variation in wave speed as they propagate.

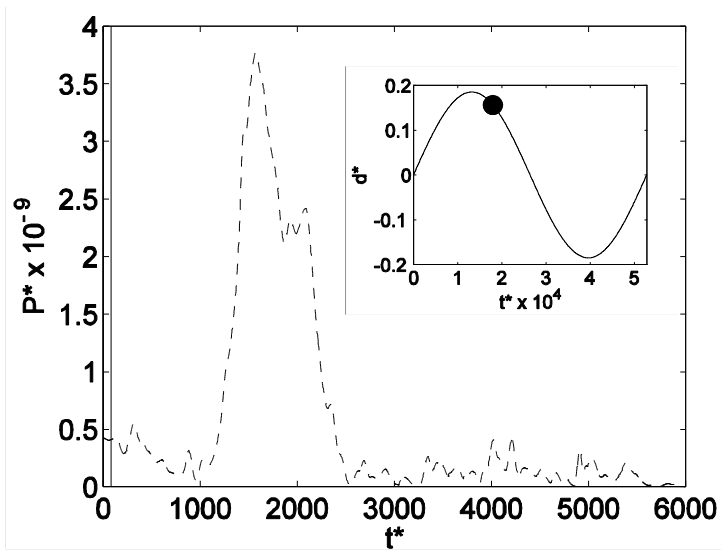

FIGURE 5. Wave shapes for pulses sent through an agitated bed. The dashed line corresponds to a sensor centered at 26 particle diameters below the free surface. The inset shows the displacement of the bed's floor when the pulse was sent.

Such pulses prove useful in diagnosing the state of an agitated bed due to their short duration relative to the period of shaking. The wave speed of a pulse can be determined at any instant of the shaking trajectory of the bed so long as particles remain in contact. This provides some insight into the current nature of the contacts and force chains in the bed as they undergo rearrangement due to agitation.

\section{REFERENCES}

1. Nesterenko, V.F., Dynamics of Heterogeneous Materials, Springer, New York, 2001.

2. Liu, C.H. and Nagel, S.R., Phys. Rev. Lett. 68, 2301-2304 (1992).

3. Coste, C., Falcon, E., and Fauve, S., Phys. Rev. E 56, 6104-6117 (1997).

4. Sen, S., Manciu, M., Sinkovits, R.S., Hurd, A.J., Granular Matter 3, 33-39 (2001).

5. Melin, S., Phys. Rev. E 49, 2353-2361 (1994).

6. Hostler, S.R. and Brennen, C.E., Proc. Mat. Res. Soc. Symp. 757, 73-78 (2003).

7. Cundall, P.A. and Strack, O.D.L., Geotechnique 29, pp. 47-65 (1979).

8. Wassgren, C.R., Vibration of Granular Material, Ph.D. Thesis, Calif. Inst. of Tech., 1997, pp. 16-37.

9. Potapov, A.V. and Campbell, C.S., Phys. Rev. Lett.77, 4760-4763 (1996). 